\title{
High variability of Panton-Valentine Leukocidine producing methicillin-resistant Staphylococcus aureus isolated from skin and soft tissue infections in the province of Bolzano
}

Richard Aschbacher', Bruno Pichon², Greta Spoladore 3 , Elisabetta Pagani', Patrizia Innocenti', Ludwig Moroder', Renate Meyer', Brigitte Ladinser', Mark Ganner'2, Robert Hill'2, Rachel Pike'2, Oswald Ganthaler ${ }^{4}$, Leonardo Pagani', Clara Larcher',Angela Kearns ${ }^{2}$

I Laboratorio Aziendale di Microbiologia e Virologia, Comprensorio Sanitario di Bolzano, Italia;

2 Microbiology Services Colindale, Health Protection Agency, London, UK;

3 Reparto di Malattie Infettive, Comprensorio Sanitario di Bolzano, Italia;

4 Laboratorio di Patologia Clinica, Comprensorio Sanitario di Brunico, Italia.

Key words: CA-MRSA, SCCmec, ACME, PVL, ST93

Elevata variabilità di Staphylococcus aureus meticillino-resistente e produttori di leucocidine di Panton-Valentine isolato da infezioni della cute e dei tessuti molli in Provincia di Bolzano

\section{SUMMARY}

Introduction. Panton-Valentine leukocindin (PVL) positive community-associated methicillin-resistant Staphylococcus aureus (CA-MRSA) isolates are widespread in many countries, with varying distribution and epidemiology. The aim of this study was to characterise the ten PVL positive MRSA isolates collected during February 2010 to January 20II from skin and soft tissue infections in the North Italian Province of Bolzano.

Methods. Accessory gene regulator (agr) typing, staphylococcal cassette chromosome (SCC) mec typing, spa typing, multi-locus sequence typing (MLST), toxin gene profiling, PCR for type I arginine catabolic mobile element (ACME) and antimicrobial resistance typing were applied to the isolates.

Results. Eight different CA-MRSA clones were identified: ST30-IVc, ST772-V, ST80-IVc, ST5-IVc, ST88-IVa, ST93IVa, ST8-IVc and the type I ACME positive ST8-IVa.

Conclusions. The high heterogeneity of PVL-positive MRSA probably reflects the introduction of different clones by international travellers or immigrants.

\section{INTRODUZIONE}

Staphylococcus aureus meticillino-resistenti (MRSA) produttori di leucocidine di PantonValentine (PVL) sono ampiamente diffusi in molti paesi, con prevalenza, distribuzione ed epidemiologia variabili (1). Sono di seguito riportati i risultati relativi alla tipizzazione dei dieci ceppi di MRSA PVL-positivi isolati in Provincia di Bolzano nel periodo da febbraio 2010 a gennaio 2011 da infezioni della cute e dei tessuti molli di pazienti d'età compresa tra 11 e 96 anni (media 50 anni); cinque dei pazienti erano stati ricoverati nel periodo dell'isolamento del ceppo o precedentemente.

\section{METODI}

Le MIC (Minime Concentrazioni Inibenti) per gli isolati di MRSA sono state determinate con il metodo di riferimento BSAC (British Society for Antimicrobial Chemotherapy) di diluizione in agar (www.bsac.org.uk/) ed interpretate secondo i criteri EUCAST (European Committee on Antimicrobial Susceptibility Testing; www.eucast.org). Gli isolati sono stati tipizzati tramite accessory gene regulator (agr) typing, staphylococcal cassette chromosome (SCC) mec typing, spa typing, multi-locus sequence typing (MLST), toxin gene profiling e PCR per arginine catabolic mobile element (ACME) di tipo I.

\section{RISULTATI}

Nel corso del periodo di studio di un anno sono stati raccolti 10 ceppi di MRSA positivi per i geni lukS-PV/lukF-PV, isolati da campioni di cute e tessuti molli provenienti da 10 pazienti senza collegamento epidemiologico fra di loro (Età media: 50 anni; range: 11-96 anni; 8 nel Comprensorio Sanitario di Bolzano e 2 nel Comprensorio Sanitario di Brunico). Tutti i nostri isolati erano positivi alla PCR per mecA con MIC per oxacilli-

\section{Corresponding author: Richard Aschbacher}

Laboratorio Aziendale di Microbiologia e Virologia - Comprensorio Sanitario di Bolzano

Via Amba Alagi, 5 - 39I00 Bolzano - Tel.: 047I 907324; Fax: 047I 27263I

E-mail: richard.aschbacher@asbz.it 
na $>4 \mathrm{mg} / \mathrm{L}$ ed avevano MIC nel range di sensibilità per clindamicina $(\leq 0.25 \mathrm{mg} / \mathrm{L})$, daptomicina $(\leq 1 \mathrm{mg} / \mathrm{L})$, acido fusidico $(\leq 0.5 \mathrm{mg} / \mathrm{L})$, linezolid $(\leq 4 \mathrm{mg} / \mathrm{L})$, mupirocina $(\leq 4 \mathrm{mg} / \mathrm{L})$, quinupristin/dalfopristina $(\leq 0.5 \mathrm{mg} / \mathrm{L})$, teicoplanina $\mathrm{e}$ vancomicina $(\leq 2 \mathrm{mg} / \mathrm{L})$. Tutte le MIC per rifampicina erano $\leq 0.03 \mathrm{mg} / \mathrm{L}$ eccetto il singolo isolato resistente (MIC $>2 \mathrm{mg} / \mathrm{L}$ ) e tutte le MIC per gentamicina erano $\leq 0.5 \mathrm{mg} / \mathrm{L}$ eccetto il singolo isolato resistente $(\mathrm{MIC}=128 \mathrm{mg} / \mathrm{L})$.
Tra i 10 isolati di MRSA PVL-positivi sono stati identificati otto differenti genotipi (Tabella 1): ST5-IVc, USA300 like ST8-IVa (ACME positivo), ST8-IVc (multi-drug resistant), South-West Pacific clone like ST30-IVc, European clone like ST80-IVc, ST88-IVa, Queensland clone like ST93-IVa (contentente un spa-repeat di 13 invece di 24 bp), e Bengal Bay clone like ST772-V (multi-drug resistant).

Tabella I. Genotipizzazione di 10 isolati di CA-MRSA PVL+ da cute e tessuti molli

\begin{tabular}{|c|c|c|c|c|c|c|c|c|c|}
\hline $\begin{array}{c}\text { Sessol } \\
\text { età }\end{array}$ & agr & MLST & spa & ACME & SCCmec & Geni tossine & Resistenze & Nome del clone & Sintomi paziente \\
\hline $\mathrm{M} / 50$ & 3 & $\begin{array}{l}\text { ST30 } \\
\text { (CC30) }\end{array}$ & t019 & - & IVc (2B) & seg, sei, pvl & CIP & $\begin{array}{l}\text { South-West- } \\
\text { Pacific like }\end{array}$ & Ulcera cutanea \\
\hline$F / 20$ & 3 & $\begin{array}{l}\text { ST30 } \\
\text { (CC30) }\end{array}$ & to19 & - & IVc (2B) & seg, sei, pvl & & $\begin{array}{l}\text { South-West- } \\
\text { Pacific like }\end{array}$ & Carbuncolo vulvare \\
\hline F/11 & 2 & $\begin{array}{l}\text { ST772 } \\
\text { (CC1) }\end{array}$ & $\mathrm{t} 345$ & - & $V(5 C)$ & $\begin{array}{c}\text { sea, sec, seg, } \\
\text { sei, pvl }\end{array}$ & $\begin{array}{l}\text { CIP, GEN, } \\
\text { ERY }\end{array}$ & Bengal-Bay like & $\begin{array}{l}\text { Secrezioni inguinali } \\
\text { purulenti }\end{array}$ \\
\hline$F / 28$ & 2 & $\begin{array}{l}\text { ST772 } \\
\text { (CC1) }\end{array}$ & $\mathrm{t} 345$ & - & $V(5 C)$ & $\begin{array}{l}\text { sea, sec, seg, } \\
\text { sei, pvl }\end{array}$ & $\begin{array}{l}\text { CIP, ERY, } \\
\text { TET }\end{array}$ & Bengal-Bay like & $\begin{array}{l}\text { Foruncoli } \\
\text { addominali }\end{array}$ \\
\hline$M / 51$ & 3 & $\begin{array}{l}\text { ST80 } \\
\text { (CC80) }\end{array}$ & t044 & - & IVc (2B) & seh, etd, pvl & CIP & European like & Foruncoli \\
\hline$M / 80$ & 2 & $\begin{array}{l}\text { ST5 } \\
\text { (CC5) }\end{array}$ & to02 & - & IVc (2B) & seg, sei, pvl & ERY & $\begin{array}{l}\text { Clone a diff. } \\
\text { mondiale }\end{array}$ & Flebite e ascesso \\
\hline$M / 61$ & 3 & $\begin{array}{c}\text { ST88 } \\
\text { (CC88) }\end{array}$ & t690 & - & IVa (2B) & $p v l$ & TET & $\begin{array}{l}\text { Raro, in vari } \\
\text { paesi }\end{array}$ & Erisipela gamba \\
\hline$M / 27$ & 3 & ST93 & $\begin{array}{l}\text { t202 } \\
\text { var. }\end{array}$ & - & IVa (2B) & $p v l$ & & Queensland like & Ascesso ferita \\
\hline F/96 & 1 & $\begin{array}{l}\text { ST8 } \\
\text { (CC8) }\end{array}$ & t008 & - & IVc (2B) & pvl & $\begin{array}{l}\text { CIP, ERY, } \\
\text { TET, RIF }\end{array}$ & Vari paesi & Infezione cutanea \\
\hline $\mathrm{M} / 74$ & 1 & $\begin{array}{l}\text { ST8 } \\
\text { (CC8) }\end{array}$ & t008 & + & IVa (2B) & $p v l$ & CIP & USA300 like & Flebite \\
\hline
\end{tabular}

Legenda: M: maschio; F: femmina; ST: sequence type; CC: clonal complex; sea, sec, seg, seh: staphylococcal enterotoxins A, I, C, H; etd: exfoliative toxin D; pvl: Panton-Valentin-Leukocidin; CIP: ciprofloxacina; GEN: gentamicina; ERY: eritromicina; TET: tetraciclina; RIF: rifampicina.

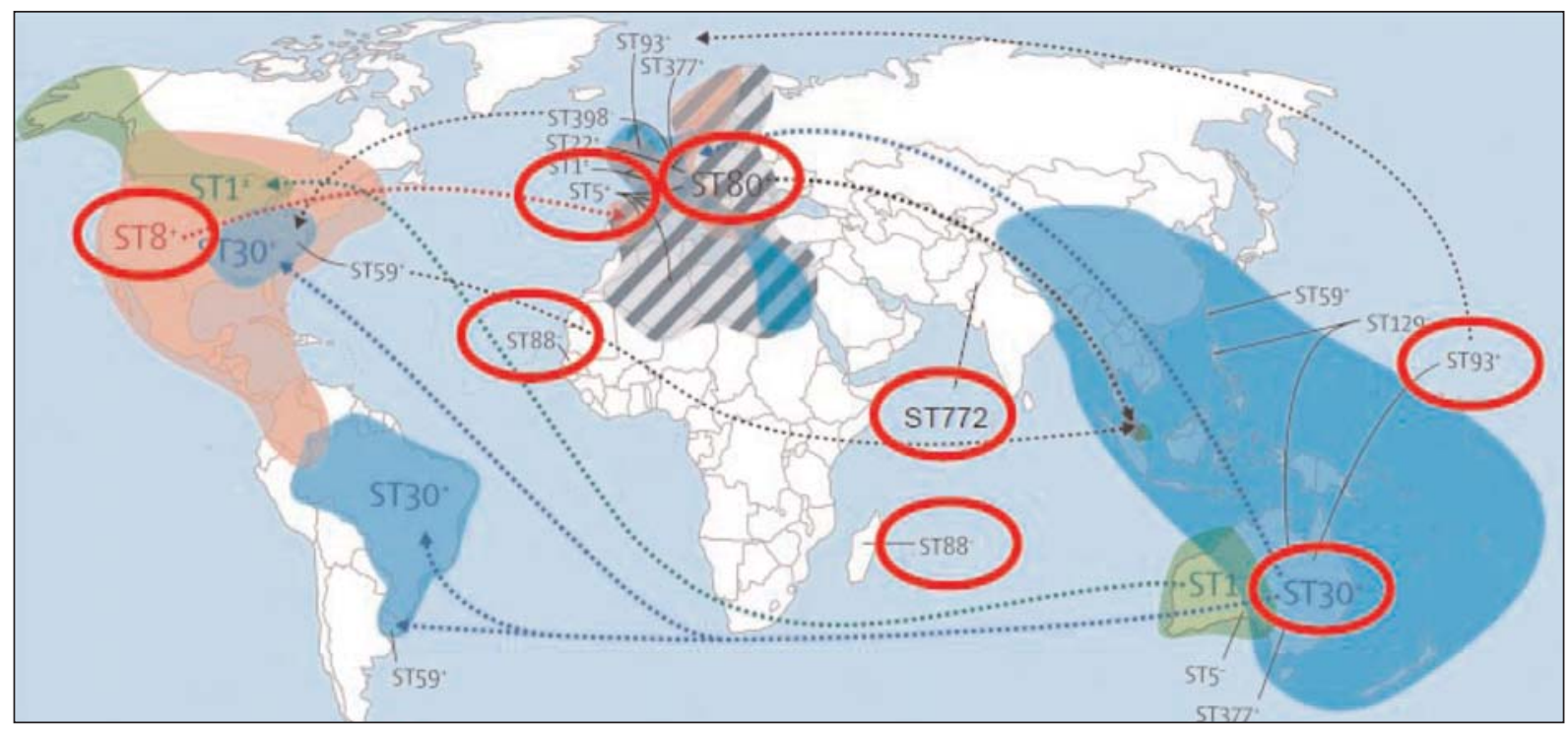

Figura I. Provenienza probabile dei ceppi MRSA PVL+ (cerchi).

\section{CONCLUSIONI}

In un numero limitato di dieci isolati di MRSA PVL-positivi da campioni cutanei abbiamo identificato dei ceppi originari da tutti i continenti (vedi immagine; 1 ; modificato). Nonostante che non era possibile raccogliere dei dati riguardanti gli spostamenti geografici dei pazienti tale dato rispecchia la globalizzazione della diffusione di CAMRSA favorita dall'immigrazione, da viaggi turistici e di lavoro e da scambi commerciali interna- 
zionali (2). Benchè il numero di isolati era limitato abbiamo trovato dei genotipi non comunemente identificati in paesi europei come ST88-IVa, ST772-V and ST93-IVa, fornendo ulteriore evidenza per la diversità di cloni presenti in Italia. In uno studio recente di CA-MRSA PVL-positivi raccolti da vari ospedali italiani e da vari tipi di infezioni gli autori hanno trovato una simile variabilità di genotipi con la identificazione di sei cloni differenti fra 18 isolati tipizzati (3). Cinque dei nostri ceppi sono stati isolati da pazienti con ricovero ospedaliero entro $i$ sei mesi precedenti o erano ricoverati al momento della raccolta dei campioni; a causa della tendenza alla diffusione intra-ospedaliera di MRSA PVL-positivi tale dato ha della implicazioni di natura igienico-sanitaria.

\section{BIBLIOGRAFIA}

1. Deleo FR et al. Community-associated methicillinresistant Staphylococcus aureus. Lancet Inf Dis 2010. 375: $1557-1568$.

2. Otter JA et al. Molecular epidemiology of communityassociated meticillin-resistant Staphylococcus aureus in Europe. Lancet Infect Dis 2010. 10: 227-239.

3. Sanchini A et al. DNA microarray-based characterisation of Panton-Valentine leukocidin-positive community-acquired methicillin-resistant Staphylococcus aureus from Italy. Eur J Clin Microbiol Infect Dis 2011. 30: 1399-1408. 